\title{
Erratum: Universality hypothesis breakdown at one-loop order [Phys. Rev. D 97, 105006 (2018)]
}

\author{
P. R. S. Carvalho®
}

Q (Received 18 May 2021; published 7 July 2021)

DOI: $10.1103 /$ PhysRevD.104.029901

In Ref. [1], there are typos in Eqs. (4)-(5), (7)-(8), (16)-(18), and (20)-(25), which must be replaced by their correct expressions, with $\Gamma_{B}^{(2)} \rightarrow q \Gamma_{B}^{(2)}$ and $\Gamma^{(2)} \rightarrow q \Gamma^{(2)}$

$$
\begin{aligned}
& \beta_{q}(u)=-\epsilon u+\frac{N+8}{6}\left(1+\frac{1}{2} \epsilon\right) q^{2} u^{2}-\frac{3 N+14}{12} q^{4} u^{3}, \\
& \gamma_{\phi, q}=\frac{N+2}{72}\left(1+\frac{5}{4} \epsilon\right) q^{4} u^{2}-\frac{(N+2)(N+8)}{864} q^{6} u^{3}, \\
& \beta_{q}(u)=-\epsilon u+\frac{N+8}{6} q^{2} u^{2}-\frac{3 N+14}{12} q^{4} u^{3}, \\
& \gamma_{\phi, q}=\frac{N+2}{72} q^{4} u^{2}-\frac{(N+2)(N+8)}{1728} q^{6} u^{3}, \\
& \gamma_{\phi^{2}, q}(u)=\frac{N+2}{6} q^{2} u-\frac{5(N+2)}{72} q^{4} u^{2}, \\
& \beta_{q}(u)=-\epsilon u+\frac{N+8}{6}\left(1-\frac{1}{2} \epsilon\right) q^{2} u^{2}-\frac{3 N+14}{12} q^{4} u^{3}, \\
& \gamma_{\phi, q}=\frac{N+2}{72}\left(1-\frac{1}{4} \epsilon+I \epsilon\right) q^{4} u^{2}-\frac{(N+2)(N+8)}{432}(1+I) q^{6} u^{3}, \\
& \alpha_{q}=\frac{(4-N)}{4(N+8)} \epsilon+\frac{(N+2)\left(N^{2}+30 N+56\right)}{4(N+8)^{3}} \epsilon^{2} \equiv \alpha, \\
& \beta_{q}=\frac{1}{2}-\frac{3}{2(N+8)} \epsilon+\frac{(N+2)(2 N+1)}{2(N+8)^{3}} \epsilon^{2} \equiv \beta, \\
& \gamma_{q}=1+\frac{(N+2)}{2(N+8)} \epsilon+\frac{(N+2)\left(N^{2}+22 N+52\right)}{4(N+8)^{3}} \epsilon^{2} \equiv \gamma, \\
& \delta_{q}=3+\epsilon+\frac{N^{2}+14 N+60}{2(N+8)^{2}} \epsilon^{2} \equiv \delta, \\
& \nu_{q}=\frac{1}{2}+\frac{(N+2)}{4(N+8)} \epsilon+\frac{(N+2)\left(N^{2}+23 N+60\right)}{8(N+8)^{3}} \epsilon^{2} \equiv \nu,
\end{aligned}
$$

Published by the American Physical Society under the terms of the Creative Commons Attribution 4.0 International license. Further distribution of this work must maintain attribution to the author(s) and the published articles title, journal citation, and DOI. 


$$
\eta_{q}=\frac{(N+2)}{2(N+8)^{2}} \epsilon^{2}\left\{1+\left[\frac{6(3 N+14)}{(N+8)^{2}}-\frac{1}{4}\right] \epsilon\right\} \equiv \eta
$$

respectively, thus meaning that the $q$-deformed critical indices are the same as their nondeformed counterparts [see Eqs. (8)-(13)]. The conclusions are now improved and more general. It was argued in Ref. [1] that the $q$ deformation of the field, while modifying its internal properties through the modification of its $q$-deformed commutation relations, would lead to the emergence of $q$-independent critical exponents. As the critical indices of Ref. [1] do not depend on $q$ at one-loop order, it was asserted that the universality hypothesis was broken down at that loop level. Now, considering the correct expressions aforementioned, Eqs. (1)-(13), the universality hypothesis is not broken down at any loop order, at least up to the loop level considered. The $q$ deformation, although it changes the internal properties of the field, is not capable of modifying its internal symmetries, namely the discrete $\phi \rightarrow-\phi$ and rotational $\mathrm{O}(N)$ ones, from which the universal critical exponents depend, among other parameters such as $d$ and $N$ for example.

P. R. S. C. would like to thank CAPES and CNPq (Brazilian funding agencies) for financial support and for the CNPq Grants No. Universal-431727/2018 and No. Produtividade 307982/2019-0.

[1] P. R. S. Carvalho, Phys. Rev. D 97, 105006 (2018). 